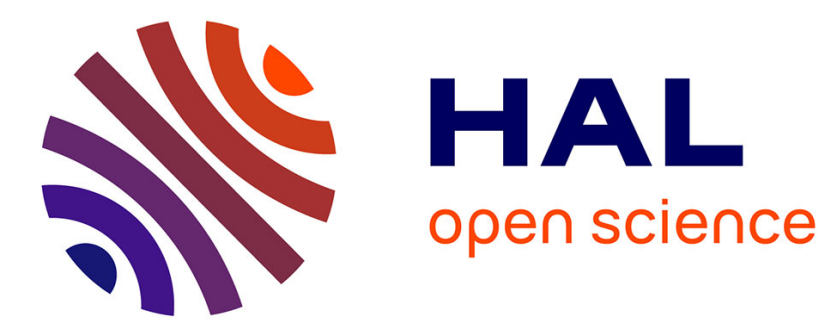

\title{
Horizons of fractional Brownian surfaces
}

Kenneth J. Falconer, Jacques Lévy Véhel

\section{To cite this version:}

Kenneth J. Falconer, Jacques Lévy Véhel. Horizons of fractional Brownian surfaces. Proceedings of the Royal Society A: Mathematical, Physical and Engineering Sciences, 2000, 456 (2001), pp.2153-2178. 10.1098/rspa.2000.0607 . inria-00581036

\section{HAL Id: inria-00581036 \\ https://hal.inria.fr/inria-00581036}

Submitted on 30 Mar 2011

HAL is a multi-disciplinary open access archive for the deposit and dissemination of scientific research documents, whether they are published or not. The documents may come from teaching and research institutions in France or abroad, or from public or private research centers.
L'archive ouverte pluridisciplinaire HAL, est destinée au dépôt et à la diffusion de documents scientifiques de niveau recherche, publiés ou non, émanant des établissements d'enseignement et de recherche français ou étrangers, des laboratoires publics ou privés. 


\title{
Horizons of fractional Brownian surfaces
}

\author{
K.J. Falconer \\ Mathematical Institute, University of St Andrews, North Haugh, St Andrews, \\ Fife, KY16 9SS, Scotland \\ and \\ J. Lévy Véhel \\ Projet Fractales, INRIA Rocquencourt, 78153 Le Chesnay Cedex, France
}

\begin{abstract}
We investigate the conjecture that the horizon of an index- $\alpha$ fractional Brownian surface has (almost surely) the same Hölder exponents as the surface itself, with corresponding relationships for fractal dimensions. We establish this formally for the usual Brownian surface (where $\alpha=\frac{1}{2}$ ), and also for other $\alpha, 0<\alpha<1$, assuming a hypothesis concerning maxima of index- $\alpha$ Brownian motion. We provide computational evidence that the conjecture is indeed true for all $\alpha$.
\end{abstract}

\section{Introduction and background}

Fractional Brownian random surfaces have found several applications in recent years in such diverse fields as computer modeling of landscapes (Mandelbrot 1982; Peitgen \& Saupe 1988), modeling of rough surfaces in physics and chemistry and engineering and in medical imaging. The parameters for such random fields need to be chosen so as to obtain the best fit to the data at hand. As an example, natural-looking landscapes typically have local Hölder exponent about 0.85 almost everywhere and fractal dimension 2.15. In some situations, an additional feature has to be taken into account: the 'horizon' defined by the surface may be relevant to modeling, and one needs to control its fractal characteristics (Mandelbrot 1982; Mandelbrot \& Van Ness 1968). For instance, it is believed that some glasses may be well modeled by fractional Brownian surfaces. Several properties of the glass, relevant both for industrial and domestic use, depend on the roughness of its horizon, which governs its behaviour under skimming lighting. More generally, fractal electromagnetism studies how waves interact with 'fractal' media (such as the sea in the case of radar imaging) (see Jaggard 1991). The horizon is then the appropriate geometrical object to consider for studying skimming waves. In imaging processes, especially medical imaging, one observes a two dimensional 'horizon' of a three dimensional object, such as a lung, and tries to infer the properties of the original object (Lundahl et al 1986). Permeability fields of a porous medium provide another example (see Addison \& Ndumu 1999).

It is conjectured that, for a 'sufficiently random and homogeneous' surface, the Hölder exponent of a horizon equals that of the surface, and that the 'fractal dimension' of the 
horizon is one less than that of the surface. In this paper we investigate this horizon conjecture for index- $\alpha$ fractional Brownian surfaces. We show that the horizon conjecture holds when $\alpha=\frac{1}{2}$, and for other values of $\alpha, 0<\alpha<1$ given a highly plausible assumption (the 'maximum property') on the form near a maximum of index- $\alpha$ fractional Brownian motion in one variable. We present direct and indirect computational evidence based on very large data sets to support the conjecture.

We first consider a function $x:[0,1]^{d} \rightarrow \mathbf{R}$, where $d$ is a positive integer, with graph graph $x \equiv\left\{(\mathbf{t}, x(\mathbf{t})): \mathbf{t} \in[0,1]^{d}\right\} \subset \mathbf{R}^{d+1}$. In particular, for $d=2$ we think of graph $x$ as a surface above the unit square $S=[0,1] \times[0,1]$ and we define the horizon function $z:[0,1] \rightarrow \mathbf{R}$ by

$$
z(t)=\sup _{0 \leq u \leq 1} x(t, u)
$$

where $\mathbf{t}=(t, u)$ in coordinate form. Thus graph $z \subset \mathbf{R}^{2}$ may be thought of as the horizon of the surface graph $x \subset \mathbf{R}^{3}$. We are interested in the relationships between the fractality of the functions $x$ and $z$ and especially between their Hölder exponents and the dimensions of the surface, graph $x$, and its horizon, graph $z$. Whilst our main concern in this paper is with index- $\alpha$ fractional Brownian surfaces, we first gives some lemmas relating to functions of a general form. We define the (lower) Hölder exponent of $x$ at $\mathbf{t}$ by

$$
\underline{H}_{x}(\mathbf{t})=\liminf _{\mathbf{v} \rightarrow \mathbf{t}} \frac{\log |x(\mathbf{v})-x(\mathbf{t})|}{\log |\mathbf{v}-\mathbf{t}|} .
$$

It is also useful to define the lower and upper approximate Hölder exponents by

$$
\underline{H}_{x}^{a p}(\mathbf{t})=\operatorname{ap} \liminf _{\mathbf{v} \rightarrow \mathbf{t}} \frac{\log |x(\mathbf{v})-x(\mathbf{t})|}{\log |\mathbf{v}-\mathbf{t}|}, \quad \bar{H}_{x}^{a p}(\mathbf{t})=\operatorname{ap} \limsup _{\mathbf{v} \rightarrow \mathbf{t}} \frac{\log |x(\mathbf{v})-x(\mathbf{t})|}{\log |\mathbf{v}-\mathbf{t}|} .
$$

Recall that the lower approximate limit is defined by ap $\liminf _{\mathbf{v} \rightarrow \mathbf{t}} f(\mathbf{v})=a$ if there exists a set $A$ of Lebesgue density 1 at $\mathbf{t}$ such that $\liminf _{\mathbf{v} \rightarrow \mathbf{t}, \mathbf{v} \in A} f(\mathbf{v})=a$, with a similar definition involving limsup for the upper approximate limit. (The set $A$ has Lebesgue density 1 at $\mathbf{t}$ if it is Lebesgue measurable and

$$
\lim _{\delta \rightarrow 0} \frac{\mathcal{L}^{d}(B(\mathbf{t}, \delta) \cap A)}{\mathcal{L}^{d}(B(\mathbf{t}, \delta))}=1
$$

where $\mathcal{L}^{d}$ is $d$-dimensional Lebesgue measure on $\mathbf{R}^{d}$ ). Clearly

$$
\underline{H}_{x}(\mathbf{t}) \leq \underline{H}_{x}^{a p}(\mathbf{t}) \leq \bar{H}_{x}^{a p}(\mathbf{t})
$$

If $\underline{H}_{x}^{a p}(\mathbf{t})=\bar{H}_{x}^{a p}(\mathbf{t})$ we say that the approximate Hölder exponent exists and write $H_{x}^{a p}(\mathbf{t})$ for the common value. We write $\operatorname{dim}_{\mathrm{H}}, \underline{\operatorname{dim}}_{\mathrm{B}}, \overline{\operatorname{dim}}_{\mathrm{B}}$ for Hausdorff, lower box and upper box dimension respectively (see Falconer 1990) for their definitions and basic properties. There are some basic lemmas that are useful in calculating dimensions of graphs. The first lemma provides an upper bound for the dimension of graph $x$, given a Hölder condition on $x$.

Lemma 1.1 Suppose that $x:[0,1]^{d} \rightarrow \mathbf{R}$ satisfies

$$
|x(\mathbf{v})-x(\mathbf{t})| \leq c|\mathbf{v}-\mathbf{t}|^{\beta}
$$

for all $\mathbf{t}, \mathbf{v} \in[0,1]^{d}$, where $0<\beta \leq 1$ and $c>0$. Then $\underline{H}_{x}(\mathbf{t}) \geq \beta$ for all $\mathbf{t} \in[0,1]^{d}$, and $\operatorname{dim}_{\mathrm{H}} \operatorname{graph} x \leq \underline{\operatorname{dim}}_{\mathrm{B}} \operatorname{graph} x \leq \overline{\operatorname{dim}}_{\mathrm{B}} \operatorname{graph} x \leq d+1-\beta$. 
Proof. See (Falconer 1990, Corollary 11.2).

The next lemma is useful for obtaining a lower bound for the dimension of a graph.

Lemma 1.2 Let $x:[0,1]^{d} \rightarrow \mathbf{R}$. Suppose that there is a number $s(1 \leq s \leq 2)$ such that for $\mathcal{L}^{d}$-almost all $\mathbf{t} \in[0,1]^{d}$ there exists $c>0$ such that

$$
\mathcal{L}^{d}\{\mathbf{v}:|\mathbf{v}-\mathbf{t}| \leq h \text { and }|x(\mathbf{v})-x(\mathbf{t})| \leq h\} \leq c h^{s}
$$

for all sufficiently small $h$. Then $\operatorname{dim}_{\mathrm{H}} \operatorname{graph} x \geq s$.

Proof. We may define a probability measure $\mu$ on graph $x$ by $\mu(A)=\mathcal{L}^{d}\left\{\mathbf{t} \in[0,1]^{d}\right.$ : $(\mathbf{t}, x(\mathbf{t})) \in A\}$. Then for $\mu$-almost all $(\mathbf{t}, x(\mathbf{t})) \in \operatorname{graph} x$,

$$
\mu(B((\mathbf{t}, x(\mathbf{t})), h)) \leq \mu(B(\mathbf{t}, h) \times[x(\mathbf{t})-h, x(\mathbf{t})+h]) \leq c h^{s}
$$

for all sufficiently small $h$, where $B(\mathbf{t}, h)$ is the ball of radius $h$ and centre $\mathbf{t}$. By Frostman's lemma $\operatorname{dim}_{\mathrm{H}} \operatorname{graph} x \geq s$.

(There is a rather weaker condition that leads to a similar lower bound for the boxdimensions, (see Falconer 1990 Corollary 11.2).) The following useful lemma shows that a Hölder condition on a surface implies a corresponding condition on its horizon, with corresponding bounds for the dimensions.

Lemma 1.3 Suppose that $x:[0,1]^{d} \rightarrow \mathbf{R}$ satisfies

$$
|x(\mathbf{v})-x(\mathbf{t})| \leq c|\mathbf{v}-\mathbf{t}|^{\beta}
$$

for all $\mathbf{t}, \mathbf{v} \in S$, where $0<\beta \leq 1$ and $c>0$. Then the horizon function $z$ given by (1.1) satisfies a Hölder condition

$$
|z(v)-z(t)| \leq c|v-t|^{\beta}
$$

for all $t, v \in[0,1]$, so in particular $\operatorname{dim}_{\mathrm{H}} \operatorname{graph} z \leq \underline{\operatorname{dim}}_{\mathrm{B}} \operatorname{graph} z \leq \overline{\operatorname{dim}}_{\mathrm{B}} \operatorname{graph} z \leq 2-\beta$ and $\underline{H}_{z}(t) \geq \beta$ for all $t \in[0,1]$.

Proof. Fix $t$ and $v$, and choose $u$ such that $z(t)=x(t, u)$ (since $u$ is continuous the supremum is attained). Then

$$
z(v) \geq x(v, u) \geq x(t, u)-c|v-t|^{\beta}=z(t)-c|v-t|^{\beta},
$$

so $z(v)-z(t) \leq c|v-t|^{\beta}$. Combining this with the symmetric inequality gives (1.6). The Hausdorff and box dimension estimates follow from Lemma 1.1, and the Hölder exponent from (1.2).

Lower bounds for dimensions of horizons are much more awkward to obtain, and we proceed to consider this problem for fractional Brownian surfaces. 


\section{Fractional Brownian fields}

A random field $X$ on the $d$-dimensional unit cube $[0,1]^{d}$ is a family of random variables $\left\{X(\mathbf{t}): \mathbf{t} \in[0,1]^{d}\right\}$ defined on some probability space. The random field $X$ is Gaussian if, for any finite set of points $\mathbf{t}_{1}, \ldots, \mathbf{t}_{n} \in[0,1]^{d}$ and scalars $\lambda_{1}, \ldots, \lambda_{n}$, the random variable $\sum_{j=1}^{n} \lambda_{j} X\left(\mathbf{t}_{j}\right)$ has Gaussian distribution. For general definitions and properties of Gaussian random fields (see Adler 1981; Geman and Horowitz 1980; Pitt 1978; Kahane 1985). We take $X$ to be a Gaussian field with zero mean, that is $\mathbf{E}(X(\mathbf{t}))=0$ for all $\mathbf{t} \in[0,1]^{d}$, where $\mathbf{E}$ denotes expectation. Such a Gaussian field is completely determined (in the sense that the joint distribution of any finite set of $X\left(\mathbf{t}_{j}\right)$ is determined) by the covariance functions

$$
\mathrm{E}\left(X\left(\mathbf{t}_{1}\right) X\left(\mathbf{t}_{2}\right)\right) \quad\left(\mathbf{t}_{1}, \mathbf{t}_{2} \in[0,1]^{d}\right),
$$

from which the variances

$$
\mathrm{E}\left(\left|X\left(\mathbf{t}_{1}\right)-X\left(\mathbf{t}_{2}\right)\right|^{2}\right)=\mathrm{E}\left(X\left(\mathbf{t}_{1}\right)^{2}\right)+\mathrm{E}\left(X\left(\mathbf{t}_{2}\right)^{2}\right)-2 \mathrm{E}\left(X\left(\mathbf{t}_{1}\right) X\left(\mathbf{t}_{2}\right)\right)
$$

may be found. The field $X$ has stationary increments if $\mathrm{E}\left(\left|X\left(\mathbf{t}_{1}\right)-X\left(\mathbf{t}_{2}\right)\right|^{2}\right)$ depends only on $\mathbf{t}_{1}-\mathbf{t}_{2}$ and has isotropic stationary increments if it depends only on $\left|\mathbf{t}_{1}-\mathbf{t}_{2}\right|$. A Gaussian field has a continuous version if there is a field with the same finite-dimensional distributions with sample paths that are a.s. continous on $[0,1]^{d}$. We specialise to the isotropic index- $\alpha$ fractional Brownian field (fBf) where $0<\alpha<1$, that is the Gaussian field $X(\mathbf{t})$ with zero mean and covariance function

$$
\mathrm{E}\left(X\left(\mathbf{t}_{1}\right) X\left(\mathbf{t}_{2}\right)\right)=\frac{1}{2}\left(\left|\mathbf{t}_{1}\right|^{2 \alpha}+\left|\mathbf{t}_{2}\right|^{2 \alpha}-\left|\mathbf{t}_{1}-\mathbf{t}_{2}\right|^{2 \alpha}\right) \quad\left(\mathbf{t}_{1}, \mathbf{t}_{2} \in[0,1]^{d}\right),
$$

(see Adler 1981). It follows easily that

$$
\mathrm{E}\left(\left|X\left(\mathbf{t}_{1}\right)-X\left(\mathbf{t}_{2}\right)\right|^{2}\right)=\left|\mathbf{t}_{1}-\mathbf{t}_{2}\right|^{2 \alpha}
$$

and that $\mathrm{E}\left(X(\mathbf{t})^{2}\right)=|\mathbf{t}|^{2 \alpha}$, so in particular $X(\mathbf{0})=0$ a.s. Thus $X$ has isotropic stationary increments and is scale invariant, in the sense that for $h \neq 0$ the Gaussian process $|h|^{-\alpha} X(h \mathbf{t})$ has the same covariance functions and thus the same distributions as $X(\mathbf{t})$. It should be emphasised that the increments are not independent in any sense unless $\alpha=\frac{1}{2}$. Index- $\alpha$ fBf has a continuous version (see Adler 1981); indeed, given $\epsilon>0$, with probability one there is a uniform Hölder inequality

$$
\left|X\left(\mathbf{t}_{1}\right)-X\left(\mathbf{t}_{2}\right)\right| \leq\left|\mathbf{t}_{1}-\mathbf{t}_{2}\right|^{\alpha-\epsilon}
$$

for all $\left|\mathbf{t}_{1}-\mathbf{t}_{2}\right|$ sufficiently small. We will always work with this continuous version of the process. It is not difficult to show, using Lemmas 1.1 and 1.2 , that $\operatorname{dim}_{\mathrm{H}} \operatorname{graph} X=$ $\underline{\operatorname{dim}}_{\mathrm{B}} \operatorname{graph} X=\overline{\operatorname{dim}}_{\mathrm{B}} \operatorname{graph} X=d+1-\alpha$ a.s. (Adler 1981; Falconer 1991).

There is an extremely useful way of regarding Gaussian fields in terms of Hilbert spaces. Let $\Omega$ be the sample space underlying a Gaussian field on $[0,1]^{d}$ and write $L^{2}(\Omega)$ for the space of zero mean random variables $X$ on $\Omega$ with $\mathrm{E}\left(X^{2}\right)<\infty$. Then $L^{2}(\Omega)$ is a real Hilbert space under the inner product given by $\mathrm{E}\left(X_{1} X_{2}\right)$ for $X_{1}, X_{2} \in L^{2}(\Omega)$. We write $H_{0}$ for the subspace $\operatorname{span}\left\{X(\mathbf{t}): \mathbf{t} \in[0,1]^{d}\right\}$ and $H$ for the closure of $H_{0}$ in $L^{2}(\Omega)$. With this Hilbert space approach, the covariances $\mathrm{E}\left(X\left(\mathbf{t}_{1}\right) X\left(\mathbf{t}_{2}\right)\right)$ are just inner products. This provides a very convenient way of representing conditional variances of the 
$X(\mathbf{t})$. For example, if $A \subset S$, then $\operatorname{var}(X(\mathbf{t}) \mid X(\mathbf{s}): \mathbf{s} \in A)$ is the Hilbert space distance from $X(\mathbf{t})$ to the subspace $K \equiv \operatorname{span}\{X(\mathbf{s}): \mathbf{s} \in A\}$ of $H$, and there is a decomposition $X(\mathbf{t})=X_{1}(\mathbf{t})+X_{2}(\mathbf{t})$, where $X_{1}(\mathbf{t}) \in \bar{K}$ and $X_{2}(\mathbf{t}) \in \bar{K}^{\perp}$. To aid calculation there is a useful integral representation of the covariances of index- $\alpha$ fBf on $[0,1]^{d}$. It is easy to check (Geman \& Horowitz 1980; Kahane 1985) that (2.1) may be written as

$$
\mathbf{E}(X(\mathbf{t}) X(\mathbf{v}))=k \int_{\mathbf{R}^{d}}|\mathbf{a}|^{-d-2 \alpha}\left(e^{i \mathbf{t} \cdot \mathbf{a}}-1\right)\left(e^{-i \mathbf{v} \cdot \mathbf{a}}-1\right) \mathrm{d} \mathbf{a},
$$

where $k$ depends only on $d$ and $\alpha$. In particular this gives that, for $\mathbf{t}_{1}, \ldots, \mathbf{t}_{n} \in \mathbf{R}^{d}$ and $\lambda_{1}, \ldots, \lambda_{n} \in \mathbf{R}$,

$$
\mathrm{E}\left(\left|\sum_{j=1}^{n} \lambda_{j} X\left(\mathbf{t}_{j}\right)\right|^{2}\right)=k \int_{\mathbf{R}^{d}}|\mathbf{a}|^{-d-2 \alpha}\left|\sum_{j=1}^{n} \lambda_{j} e^{i \mathbf{t}_{j} \cdot \mathbf{a}}\right|^{2} \mathrm{~d} \mathbf{a},
$$

provided that $\sum_{j=1}^{n} \lambda_{j}=0$. This integral form provides a very useful way of calculating conditional variances of increments of the $X(\mathbf{t})$. For $E$ an ellipsoid in $\mathbf{R}^{d}$ and $\gamma>1$, write $E_{\gamma}$ for the homothetic (similar and similarly situated) ellipsoid with the same centre as $E$ obtained by scaling $E$ by a factor $\lambda$ about its centre. For $T \subset \mathbf{R}^{d}$ we write $\mathcal{F}_{T}$ for the sigma field $\sigma\left(X\left(\mathbf{t}_{1}\right)-X\left(\mathbf{t}_{2}\right): \mathbf{t}_{1}, \mathbf{t}_{2} \in T\right)$ generated by the differences of $X$ at pairs of points in $T$.

Proposition 2.1 Let $E \subset \mathbf{R}^{d}$ be an ellipsoid with semi-axis lengths $0<s_{1} \leq \ldots \leq s_{d}$ and let $E_{\gamma}$ be defined as above for some $\gamma>1$. If $V \subset E$ and $T \subset E_{\gamma}^{c}$ (the complement of $\left.E_{\gamma}\right)$, and $\mathbf{v} \in V$ and $\mathbf{t} \in T$, then

$$
\operatorname{var} \equiv \operatorname{var}\left(X(\mathbf{v})-X(\mathbf{t}) \mid \sigma\left(\mathcal{F}_{T}, \mathcal{F}_{V}\right)\right) \geq c s_{1}^{d+2 \alpha}\left(s_{1} \ldots s_{d}\right)^{-1}
$$

and hence, for all $\rho_{1}<\rho_{2}$,

$$
\mathrm{P}\left(\rho_{1}<X(\mathbf{v})-X(\mathbf{t}) \leq \rho_{2} \mid \sigma\left(\mathcal{F}_{T}, \mathcal{F}_{V}\right)\right) \leq c_{0}\left|\rho_{2}-\rho_{1}\right| s_{1}^{-d / 2-\alpha}\left(s_{1} \ldots s_{d}\right)^{1 / 2},
$$

where $c$ and $c_{0}$ depend only on $d, \alpha$ and $\gamma$.

Proof. Working in the Hilbert space setting, var is the square of the Hilbert space distance of $X(\mathbf{v})-X(\mathbf{t})$ from the subspace defined by the conditioning; thus

$$
\begin{aligned}
\operatorname{var}=\inf \mathrm{E}(\mid X(\mathbf{v})-X(\mathbf{t}) & +\sum_{j=1}^{m} \lambda_{j}\left(X\left(\mathbf{v}_{j}\right)-X(\mathbf{v})\right) \\
& \left.+\sum_{j=1}^{m^{\prime}} \eta_{j}\left(X\left(\mathbf{t}_{j}\right)-X(\mathbf{t})\right): \mathbf{v}_{j} \in V,\left.\mathbf{t}_{j} \in T\right|^{2}\right)
\end{aligned}
$$

where the infimum is over all $\lambda_{1}, \ldots, \lambda_{m}, \eta_{1}, \ldots, \eta_{m^{\prime}} \in \mathbf{R}, \mathbf{v}_{1}, \ldots, \mathbf{v}_{m} \in V$ and $\mathbf{t}_{1}, \ldots, \mathbf{t}_{m^{\prime}} \in$ $V$. By redefining the $\lambda_{j}, \eta_{j}$ by taking appropriate linear combinations in the sums, we get that

$$
\operatorname{var} \geq \inf \mathrm{E}\left(\left|\sum_{j} \lambda_{j} X\left(\mathbf{v}_{j}\right)-\sum_{j} \eta_{j} X\left(\mathbf{t}_{j}\right): \mathbf{v}_{j} \in V, \mathbf{t}_{j} \in T, \sum_{j} \lambda_{j}=\sum_{j} \eta_{j}=1\right|^{2}\right) .
$$


Since $\sum_{j} \lambda_{j}-\sum_{j} \eta_{j}=0$, the integral representation (2.5) gives

$$
\begin{aligned}
\operatorname{var} \geq \inf k\left\{\int \frac{\left|\sum_{j} \lambda_{j} e^{i \mathbf{v}_{j} \cdot \mathbf{a}}-\sum_{j} \eta_{j} e^{i \mathbf{t}_{j} \cdot \mathbf{a}}\right|^{2}}{|\mathbf{a}|^{d+2 \alpha}}\right. & \text { a } \\
& \left.: \mathbf{v}_{j} \in V, \mathbf{t}_{j} \in T, \sum_{j} \lambda_{j}=\sum_{j} \eta_{j}=1\right\} .
\end{aligned}
$$

Defining the Fourier transform $\hat{g}$ of a 'well-behaved' function $g$ on $\mathbf{R}^{d}$ by

$$
\hat{g}(\mathbf{b})=\int_{-\infty}^{\infty} e^{i \mathbf{b} \cdot \mathbf{w}} g(\mathbf{w}) \mathrm{d} \mathbf{w}
$$

in the usual way, the inversion formula gives

$$
g(\mathbf{w})=(2 \pi)^{-d} \int_{-\infty}^{\infty} e^{-i \mathbf{b} \cdot \mathbf{w}} \hat{g}(\mathbf{b}) \mathrm{d} \mathbf{b} .
$$

We take $g$ to be a $C^{\infty}$ 'bump function', with $g(\mathbf{w})=1$ for $\mathbf{w} \in B(\mathbf{0}, 1)$ and $g(\mathbf{w})=0$ for $\mathbf{w} \notin B(\mathbf{0}, \gamma)$. Let $\mathbf{p} \in \mathbf{R}^{d}$ be the centre of the ellipsoid $E$ and let $\phi$ be a non-singular self-adjoint linear operator on $\mathbf{R}^{d}$ that maps the unit ball onto the translated ellipsoid $E-\mathbf{p}$; thus $E=\phi(B(\mathbf{0}, 1))+\mathbf{p}$ and $E_{\gamma}=\phi(B(\mathbf{0}, \gamma))+\mathbf{p}$. With $\sum_{j} \lambda_{j}=\sum_{j} \eta_{j}=1$, it follows, using the definitions of $\phi$ and $g$, that

$$
\begin{aligned}
1 & =\sum_{j} \lambda_{j} g\left(\phi^{-1}\left(\mathbf{v}_{j}-\mathbf{p}\right)\right)-\sum_{j} \eta_{j} g\left(\phi^{-1}\left(\mathbf{t}_{j}-\mathbf{p}\right)\right) \\
& =(2 \pi)^{-d} \int\left(\sum_{j} \lambda_{j} e^{i \mathbf{b} \cdot \phi^{-1}\left(\mathbf{p}-\mathbf{v}_{j}\right)}-\sum_{j} \eta_{j} e^{i \mathbf{b} \cdot \phi^{-1}\left(\mathbf{p}-\mathbf{t}_{j}\right)}\right) \hat{g}(\mathbf{b}) \mathrm{d} \mathbf{b} \\
& =(2 \pi)^{-d} \int\left(\sum_{j} \lambda_{j} e^{i \mathbf{a} \cdot\left(\mathbf{p}-\mathbf{v}_{j}\right)}-\sum_{j} \eta_{j} e^{i \mathbf{a} \cdot\left(\mathbf{p}-\mathbf{t}_{j}\right)}\right) \hat{g}(\phi(\mathbf{a}))|\operatorname{det} \phi| \mathrm{d} \mathbf{a}
\end{aligned}
$$

writing $\mathbf{b}=\phi(\mathbf{a})$ and using the self-adjointness of $\phi$. Thus by Cauchy's inequality,

$$
\begin{aligned}
1 & \leq(2 \pi)^{-2 d} \int \frac{\left|e^{i \mathbf{a} \cdot \mathbf{p}}\right|^{2}\left|\sum_{j} \lambda_{j} e^{-i \mathbf{a} \cdot \mathbf{v}_{j}}-\sum_{j} \eta_{j} e^{-i \mathbf{a} \cdot \mathbf{t}_{j}}\right|^{2}}{|\mathbf{a}|^{d+2 \alpha}} \mathrm{d} \mathbf{a} \int \hat{g}(\phi(\mathbf{a}))^{2}|\mathbf{a}|^{d+2 \alpha}|\operatorname{det} \phi|^{2} \mathrm{~d} \mathbf{a} \\
& \leq(2 \pi)^{-2 d} \int \frac{\left|\sum_{j} \lambda_{j} e^{i \mathbf{a} \cdot \mathbf{v}_{j}}-\sum_{j} \eta_{j} e^{i \mathbf{a} \cdot \mathbf{t}_{j}}\right|^{2}}{|\mathbf{a}|^{d+2 \alpha}} \mathrm{d} \mathbf{a} \int \hat{g}(\mathbf{b})^{2}\left|\phi^{-1}(\mathbf{b})\right|^{d+2 \alpha} \mathrm{d} \mathbf{b}|\operatorname{det} \phi| \\
& \leq(2 \pi)^{-2 d} \int \frac{\left|\sum_{j} \lambda_{j} e^{i \mathbf{a} \cdot \mathbf{v}_{j}}-\sum_{j} \eta_{j} e^{i \mathbf{a} \cdot \mathbf{t}_{j}}\right|^{2}}{|\mathbf{a}|^{d+2 \alpha}} \mathrm{d} \mathbf{a} \int \hat{g}(\mathbf{b})^{2}|\mathbf{b}|^{d+2 \alpha} \mathrm{d} \mathbf{b} s_{1}^{-d-2 \alpha}\left(s_{1} \ldots s_{d}\right),
\end{aligned}
$$

as $\operatorname{det} \phi=s_{1} \ldots s_{d}$ and $\left\|\phi^{-1}\right\|=s_{1}^{-1}$. Since $\hat{g}(\mathbf{b})$ is continuous and rapidly decreasing, the last integral is finite, so combining this estimate with (2.8) gives (2.6). Inequality (2.7) follows on noting that, if $W$ is Gaussian with mean $\mu$ and variance $\sigma^{2}$, then

$$
\mathrm{P}\left(\rho_{1}<W \leq \rho_{2}\right)=(2 \pi)^{-1 / 2} \sigma^{-1} \int_{\rho_{1}}^{\rho_{2}} \exp \left(-(s-\mu) / 2 \sigma^{2}\right) \mathrm{d} s \leq(2 \pi)^{-1 / 2} \sigma^{-1}\left|\rho_{2}-\rho_{1}\right| .
$$




\section{The maximum property for $\mathrm{fBm}$}

In this section we briefly consider index- $\alpha$ fractional Brownian motion (fBm) $X:[0,1] \rightarrow$ $\mathbf{R}$, that is the index- $\alpha$ fBf defined by (2.1) with $d=1$. Then $X$ has a continuous version which a.s. attains its maximum at a unique point of $(0,1)$. We say that index- $\alpha \mathrm{fBm} X$ on $[0,1]$ satisfies the maximum property if, conditional on $X$ attaining its maximum at $t_{m} \in(0,1)$, for all $\epsilon>0$ there exists a.s. $\delta>0$ such that

$$
X(t) \leq X\left(t_{m}\right)-\left|t_{m}-t\right|^{\alpha+\epsilon} \text { for all } t \in[0,1] \text { with }\left|t_{m}-t\right| \leq \delta .
$$

Of course, the opposite inequality, that for all $\epsilon>0$

$$
X(t) \geq X\left(t_{m}\right)-\left|t_{m}-t\right|^{\alpha-\epsilon}
$$

for all $t$ close to $t_{m}$, follows from the a.s. Hölder condition (2.3). Thus $X$ has the maximum property if its sample paths fall away from their maximum at a rate with power law exponent close to $\alpha$. It should be emphasised that the maximum property is defined in terms of fractional Brownian motion on the real interval and not in terms of fractional Brownian fields on $\mathbf{R}^{2}$, although this is where our applications lie.

Proposition 3.1 Usual Brownian motion, that is index $-\frac{1}{2} f B m$, has the maximum property.

Proof. This fact, stated for example in (Barlow \& Perkins 1984), follows from the decomposition theorem (Itô \& McKean 1974; Williams 1974) which identifies Brownian motion conditioned on a maximum at 0 with the 3 -dimensional Bessel process (that is the radial component of Brownian motion in $\mathbf{R}^{3}$ ).

The following maximum conjecture seems extremely likely, but it does not appear to have been established rigorously.

Conjecture 3.2 For all $0<\alpha<1$, index- $\alpha$ fBm has the maximum property.

This conjecture holds in an 'approximate' sense: conditional on $X$ attaining its maximum at $t$, we have a.s. that for all $\epsilon>0$

$$
\operatorname{ap} \limsup _{t \rightarrow t}\left(X(t)-X\left(t_{m}\right)+\left|t_{m}-t\right|^{\alpha+\epsilon}\right) \leq 0
$$

(see Geman \& Horowitz 1980, Theorem 30.4), but this is not sufficient for our arguments.

\section{Probabilistic estimates}

From now on, we take $d=2$ and work with the index- $\alpha$ fractional Brownian field $X: S \rightarrow$ $\mathbf{R}$ where $S$ is the unit square $[0,1] \times[0,1]$. In this case we refer to graph $X$ as the index- $\alpha$ fractional Brownian surface. In this section we estimate some conditional variances and probabilities of $X$ preparatory to our calculations on the fractality of the horizon of $X$. We use the vector and coordinate forms $\mathbf{t}=(t, u), \mathbf{v}=(v, w), \mathbf{t}_{1}=\left(t_{1}, u_{1}\right)$ etc. for points in $\mathbf{R}^{2}$. We introduce sigma-fields generated by the random variables $X(t, u)$ on certain 
subsets of the square $S$. For $t \in[0,1]$ let $\mathcal{F}_{t}=\sigma\left(X\left(t, u_{1}\right)-X\left(t, u_{2}\right): u_{1}, u_{2} \in[0,1]\right)$, and for $(v, u) \in S$ and $r>0$ let $\mathcal{F}_{(v, u), r}=\sigma(X(v, w)-X(v, u): w \in[0,1],|w-u| \leq r)$. Thus $\mathcal{F}_{t}$ determines $X$ along a section with first coordinate $t$, and $\mathcal{F}_{(v, u), r}$ determines $X$ along a section of length $2 r$ with midpoint $(v, u)$, in both cases to within a vertical displacement.

Proposition 4.1 For all $r>0$ and all pairs of points $(t, u),(v, u) \in S$ with $0<|v-t| \leq r$, we have, almost surely,

$$
\operatorname{var}\left(X(v, u)-X(t, u) \mid \sigma\left(\mathcal{F}_{t}, \mathcal{F}_{(v, u), r}\right)\right) \geq c|v-t|^{1+2 \alpha} r^{-1}
$$

and for all $\rho_{1}<\rho_{2}$,

$$
\mathrm{P}\left(\rho_{1}<X(v, u)-X(t, u) \leq \rho_{2} \mid \sigma\left(\mathcal{F}_{t}, \mathcal{F}_{(v, u), r}\right)\right) \leq c_{0}\left|\rho_{2}-\rho_{1}\right||v-t|^{-1 / 2-\alpha} r^{1 / 2},
$$

where $c$ and $c_{0}$ depend only on $d$ and $\alpha$.

Proof. We apply Proposition 2.1, taking $E$ to be the ellipse with centre $(u, v)$ and semiaxes parallel to the coordinate axes, of lengths $\frac{1}{3}|t-v|$ and $r$, and taking $\gamma=2$. Then $E$ contains the interval $[(v, u-r),(v, u+r)]$ and $E_{\gamma}$ is disjoint from the line $\{(t, w): t \in \mathbf{R}\}$, so the result is immediate from Proposition 2.1

The (random) horizon function of the index- $\alpha$ fBf $X(t, u)$ is given by

$$
Z(t)=\sup _{0 \leq u \leq 1} X(t, u) \quad(0 \leq t \leq 1)
$$

Since $X$ is almost surely continuous, for each $t \in[0,1]$ we can define the random variable $U_{t}$ by

$$
U_{t}=\inf \{u \in[0,1]: X(u, t)=Z(t)\},
$$

so $U_{t}$ is $\mathcal{F}_{t}$-measurable. (In fact $X(t, u)$ a.s. attains a unique maximum at $u=U_{t} \in(0,1)$, with $Z(t)=X\left(t, U_{t}\right)$, see Section 5.) For $r>0$ and $t, v \in[0,1]$, we define the random variable

$$
Z_{t}^{r}(v)=\sup \left\{X(v, u): u \in[0,1] \text { and }\left|u-U_{t}\right| \leq r\right\}
$$

Then $Z_{t}^{r}(v)$ gives the maximum of $X(v, u)$ over a restricted interval of $u$ close to $U_{t}$.

Proposition 4.2 For all $r>0, \rho_{1}<\rho_{2}$ and $t, v \in[0,1]$ with $0<|v-t| \leq r$, we have

$$
\mathrm{P}\left(\rho_{1}<Z_{t}^{r}(v)-Z(t) \leq \rho_{2}\right) \leq c_{0}\left|\rho_{2}-\rho_{1}\right||v-t|^{-1 / 2-\alpha} r^{1 / 2},
$$

where $c_{0}$ depends only on $\alpha$.

Proof. For $(v, u) \in S$ define the random variable

$$
Z(v, u)=\sup \{X(v, w):|w-u| \leq r \text { and }(v, w) \in S\}
$$

Since $Z(t)-X(t, u)$ and $Z(v, u)-X(v, u)$ are $\mathcal{F}_{t}$-measurable and $\mathcal{F}_{(v, u), r}$-measurable respectively, and therefore are both $\sigma\left(\mathcal{F}_{t}, \mathcal{F}_{(v, u), r}\right)$-measurable, Proposition 4.1 implies that for all $t \in[0,1]$ and $(v, u) \in S$,

$$
\mathrm{P}\left(\rho_{1}<Z(v, u)-Z(t) \leq \rho_{2} \mid \sigma\left(\mathcal{F}_{t}, \mathcal{F}_{(v, u), r}\right)\right) \leq c_{0}\left|\rho_{2}-\rho_{1}\right||v-t|^{-1 / 2-\alpha} r^{1 / 2},
$$


a.s.. For $n=1,2, \ldots$ define the random variables

$$
U_{t}^{n}=\frac{1}{n}\left[n U_{t}\right]
$$

where [ ] denotes 'the integer part of'. Then $U_{t}^{n}$ is $\mathcal{F}_{t}$-measurable and finite valued and gives a discrete approximation to $U_{t}$ (we need to invoke this discretisation for step (4.6) below). Since $\sigma\left(U_{t}^{n}\right) \subset \sigma\left(\mathcal{F}_{t}, \mathcal{F}_{(v, u), r}\right)$, the tower property for conditional probabilities gives, for each $i=1, \ldots, n$,

$$
\mathrm{P}\left(\rho_{1}<Z\left(v, \frac{i}{n}\right)-Z(t) \leq \rho_{2} \mid U_{t}^{n}\right) \leq c_{0}\left|\rho_{2}-\rho_{1}\right||v-t|^{-1 / 2-\alpha} r^{1 / 2} .
$$

Then

$$
\begin{aligned}
\mathrm{P}\left(\rho_{1}<Z\left(v, U_{t}^{n}\right)-Z(t) \leq \rho_{2}\right) & =\sum_{i=0}^{n-1} \mathrm{P}\left(\rho_{1}<Z\left(v, \frac{i}{n}\right)-Z(t) \leq \rho_{2} \mid U_{t}^{n}=\frac{i}{n}\right) \mathrm{P}\left(U_{t}^{n}=\frac{i}{n}\right) \\
& \leq c_{0}\left|\rho_{2}-\rho_{1}\right||v-t|^{-1 / 2-\alpha} r^{1 / 2}
\end{aligned}
$$

Since $X$ is a.s. continuous, $Z\left(v, U_{t}^{n}\right)-Z(t)$ converges a.s. to $Z\left(v, U_{t}\right)-Z(t)$ as $n \rightarrow \infty$, and hence converges in distribution, so (4.5) follows from (4.6).

Fix $\eta>0$ and define, for $t, v \in[0,1]$,

$$
Z_{t}(v)=\sup \left\{X(v, u): u \in[0,1] \text { and }\left|u-U_{t}\right| \leq|v-t|^{1-\eta}\right\} .
$$

Thus $Z_{t}(v)$ is just $Z_{t}^{r}(v)$ of (4.4) taking $r=|v-t|^{1-\eta} \geq|v-t|$.

Corollary 4.3 For all $t \in[0,1]$ and $0<\eta<1-\alpha$, we have a.s. that

$$
\mathcal{L}\left(v \in[0,1]:|v-t| \leq h \text { and }\left|Z_{t}(v)-Z(t)\right| \leq h\right) \leq h^{2-\alpha-\eta}
$$

and

$$
\operatorname{ap} \limsup _{v \rightarrow t} \frac{\log \left|Z_{t}(v)-Z(t)\right|}{\log |v-t|} \leq \alpha
$$

for all sufficiently small $h$.

Proof. Setting $r=|v-t|^{1-\eta}$ in Proposition 4.2 and replacing $Z_{t}^{r}$ by $Z_{t}$, noting (4.4) and (4.7), gives

$$
\mathrm{P}\left(\rho_{1}<Z_{t}(v)-Z(t) \leq \rho_{2}\right) \leq c_{0}\left|\rho_{2}-\rho_{1}\right||v-t|^{-\alpha-\eta / 2} .
$$

For $t \in[0,1]$,

$$
\begin{aligned}
\mathrm{E}\left(\mathcal{L}\left(v:|v-t| \leq h \text { and }\left|Z_{t}(v)-Z(t)\right| \leq h\right)\right) & \leq \int_{t-h}^{t+h} \mathrm{P}\left(\left|Z_{t}(v)-Z(t)\right| \leq h\right) \mathrm{d} v \\
& \leq 2 c_{0} h \int_{t-h}^{t+h}|v-t|^{-\alpha-\eta / 2} \mathrm{~d} v \\
& =4 c_{0}(1-\alpha-\eta / 2)^{-1} h^{2-\alpha-\eta / 2} .
\end{aligned}
$$

Thus for each $t \in[0,1]$, by applying (4.11) with $h=2^{-k}(k=1,2, \ldots)$ and using the Borel-Cantelli lemma, we get a.s. that (4.8) holds for all sufficiently small $h$. Again using 
(4.10), we have

$$
\begin{aligned}
\mathrm{E}\left(\mathcal { L } \left(v:|v-t| \leq h \text { and }\left|Z_{t}(v)-Z(t)\right|\right.\right. & \left.\left.\leq|v-t|^{\alpha+\eta}\right)\right) \\
& \leq \int_{t-h}^{t+h} \mathrm{P}\left(\left|Z_{t}(v)-Z(t)\right| \leq|v-t|^{\alpha+\eta}\right) \mathrm{d} v \\
& \leq 2 c_{0} \int_{t-h}^{t+h}|v-t|^{\eta / 2} \mathrm{~d} v \\
& =4 c_{0}(1+\eta / 2)^{-1} h^{1+\eta / 2},
\end{aligned}
$$

so another application of the Borel-Cantelli lemma gives

$$
\frac{1}{2 h} \mathcal{L}\left(v \in[0,1]:|v-t| \leq h \text { and }\left|Z_{t}(v)-Z(t)\right| \leq|v-t|^{\alpha+\eta}\right) \leq h^{\eta / 4}
$$

a.s. for sufficiently small $h$, from which (4.9) follows using (1.3).

\section{Horizons of fractional Brownian surfaces}

Recall that the horizon of the index- $\alpha$ fBf $X$ on $S=[0,1] \times[0,1]$ is the random function $Z$ on $[0,1]$ given by

$$
Z(t)=\sup _{0 \leq u \leq 1} X(t, u) .
$$

Since $X$ satisfies the Hölder condition (2.3) a.s., Lemma 1.3 implies that a.s. $\underline{H}_{Z}(t) \geq$ $\alpha$ for all $t \in[0,1]$ and that $\operatorname{dim}_{\mathrm{H}} \operatorname{graph} Z \leq \underline{\operatorname{dim}}_{\mathrm{B}} \operatorname{graph} Z \leq \overline{\operatorname{dim}}_{\mathrm{B}} \operatorname{graph} Z \leq 2-\alpha$. Estimates in the other direction are more awkward. It was shown in (Falconer 1991) that $H_{Z}(t) \leq \alpha+\frac{1}{2}$ and $1 \frac{1}{2}-\alpha \leq \operatorname{dim}_{\mathrm{H}}$ graph $Z$ almost surely. Here we show that $\operatorname{dim}_{\mathrm{H}} \operatorname{graph} Z=\underline{\operatorname{dim}}_{\mathrm{B}} \operatorname{graph} Z=\overline{\operatorname{dim}}_{\mathrm{B}} \operatorname{graph} Z=2-\alpha$ almost surely if $\alpha=\frac{1}{2}$, and also for all $0<\alpha<1$ if we assume the maximum Conjecture 3.2 for index- $\alpha$ fractional Brownian motion on the line. Our strategy is to show that, for each $t$, we have $Z(v)=Z_{t}(v)$, for all $v$ sufficiently close to $t$, see (4.4), and then apply Lemma 1.2 using the estimate of Corollary 4.3 to obtain the dimensions and Hölder exponents. With $U_{t}$ the value of $u$ at which the maximum $\sup _{0 \leq u \leq 1} X(t, u)=Z(t)$ is attained (4.3), we consider how $U_{t}$ varies with $t$.

Proposition 5.1 Assume that index- $\alpha$ fBm has the maximum property. Let $\eta>0$. Then for all $t \in[0,1]$, there exists a.s. $\delta>0$ such that, for all $v \in[0,1]$ with $|v-t|<\delta$,

$$
\left|U_{v}-U_{t}\right| \leq|v-t|^{1-\eta}
$$

Proof. Choose $0<\epsilon<\alpha$ such that

$$
1-\eta<(\alpha-\epsilon) /(\alpha+\epsilon) .
$$

Index- $\alpha$ fBf satisfies an a.s. uniform Hölder condition (2.3) which may be invoked in the form: there exists a.s. a number $\delta_{1}>0$ such that

$$
|X(t, u)-X(v, w)| \leq \frac{1}{3}|(t, u)-(v, w)|^{\alpha-\epsilon}
$$

for all $(t, u),(v, w) \in S$ with $|(t, u)-(v, w)| \leq \delta_{1}$. Fix $t \in[0,1]$ for the remainder of the proof. The process $X(t, \cdot)-X(t, 0):[0,1] \rightarrow \mathbf{R}$ is index- $\alpha \mathrm{fBm}$, being a section of index- $\alpha$ 
fBf, so, using the maximum property $(3.1)$, a.s. $X(t, u)$ attains a unique maximum at $u=U_{t} \in(0,1)$, such that for some $\delta_{2}>0$

$$
X(t, u) \leq X\left(t, U_{t}\right)-\left|u-U_{t}\right|^{\alpha+\epsilon}
$$

if $\left|u-U_{t}\right| \leq \delta_{2}$. Using the uniqueness of the maximum and a continuity argument, this implies that a.s. there is some (random) $\lambda>0$ such that

$$
X(t, u) \leq X\left(t, U_{t}\right)-\min \left\{\left|u-U_{t}\right|^{\alpha+\epsilon}, \lambda\right\} \text { for all } u \in[0,1] .
$$

Set $\delta=\min \left\{\delta_{1}, \lambda^{1 /(\alpha-\epsilon)}\right\}$. If

$$
|v-t|<\delta \leq \lambda^{1 /(\alpha-\epsilon)}
$$

then by (5.4)

$$
X\left(v, U_{t}\right) \geq X\left(t, U_{t}\right)-\frac{1}{3}|v-t|^{\alpha-\epsilon} .
$$

On the other hand, suppose $(v, u)$ is such that

$$
|v-t|<\delta \text { and }\left|u-U_{t}\right| \geq|v-t|^{1-\eta} .
$$

Then by (5.4),(5.5),(5.8), (5.6) and (5.3),

$$
\begin{aligned}
X(v, u) & \leq X(t, u)+\frac{1}{3}|v-t|^{\alpha-\epsilon} \\
& \leq X\left(t, U_{t}\right)-\min \left\{\left|u-U_{t}\right|^{\alpha+\epsilon}, \lambda\right\}+\frac{1}{3}|v-t|^{\alpha-\epsilon} \\
& \leq X\left(t, U_{t}\right)-\min \left\{|v-t|^{(\alpha+\epsilon)(1-\eta)},|v-t|^{\alpha-\epsilon}\right\}+\frac{1}{3}|v-t|^{\alpha-\epsilon} \\
& \leq X\left(t, U_{t}\right)-\frac{2}{3}|v-t|^{\alpha-\epsilon} .
\end{aligned}
$$

Comparing with (5.7) it follows that if $|v-t|<\delta$ the supremum $\sup _{0 \leq u \leq 1} X(v, u)$ cannot be attained by $u$ such that $\left|u-U_{t}\right| \geq|v-t|^{1-\eta}$, and the result follows.

We can now conclude that $Z(v)=Z_{t}(v)$ if $v$ is close to $t$.

Proposition 5.2 Assume that index- $\alpha$ fBm has the maximum property. Then for all $t \in[0,1]$, there exists a.s. $\delta>0$ such that, for all $v \in[0,1]$ with $|v-t|<\delta$,

$$
Z(v)=Z_{t}(v)
$$

Proof. This is immediate from Proposition 5.1 and the definitions (4.3) and (4.7) of $U_{t}$ and $Z_{t}(v)$.

We now combine our results to obtain the fractal properties of the horizon.

Theorem 5.3 Assume that index- $\alpha f B m$ has the maximum property. Writing $Z(t)$ for the horizon function of the index- $\alpha$ fBf $X(t, u)$, we have that $\operatorname{dim}_{\mathrm{H}} \operatorname{graph} Z=\underline{\operatorname{dim}}_{\mathrm{B}} \operatorname{graph} Z=$ $\overline{\operatorname{dim}}_{\mathrm{B}} \operatorname{graph} Z=2-\alpha$ a.s..

Proof. By Corollory 4.3 and Proposition 5.2 we have for all $\eta>0$ and $t \in[0,1]$, that a.s.

$$
\mathcal{L}(v:|v-t| \leq h \text { and }|Z(v)-Z(t)| \leq h) \leq h^{2-\alpha-\eta}
$$

for $h$ sufficiently small. Hence with probability one, for $\mathcal{L}$-almost all $t \in[0,1],(5.10)$ holds for all $0<h<h(t)$ for some $h(t)>0$. Since $\eta>0$ is arbitrary, Lemma 1.2 gives $\operatorname{dim}_{\mathrm{H}} \operatorname{graph} Z \geq 2-\alpha$ a.s.. Since $X$ satisfies an almost sure uniform Hölder condition (2.3), the opposite inequality follows from Lemma 1.3.

We can obtain Hölder exponents in a similar way. 
Theorem 5.4 Assume that index- $\alpha f B m$ has the maximum property. Writing $Z(t)$ for the horizon function of the index- $\alpha f B f X(t, u)$, we have that a.s.,

$$
\underline{H}_{Z}(t)=H_{Z}^{a p}(t)=\alpha
$$

for almost all $t \in[0,1]$.

Proof. Lemma 1.3 and (2.3)imply that a.s.

$$
\alpha \leq \underline{H}_{Z}(t) \leq \underline{H}_{Z}^{a p}(t) \leq \bar{H}_{Z}^{a p}(t)
$$

for all $t$. Proposition 5.2 along with (4.9) gives that for all $t \in[0,1]$, we have

$$
\operatorname{ap} \limsup _{v \rightarrow t} \frac{\log |Z(v)-Z(t)|}{\log |v-t|} \leq \alpha
$$

a.s., that is $\bar{H}_{Z}^{a p}(t) \leq \alpha$ a.s., and Fubini's theorem gives the conclusion.

Corollary 5.5 Let $Z$ be the horizon function of the usual (index- $\frac{1}{2}$ ) fBf. Then, with probability one, $\underline{H}_{Z}(t)=H_{Z}^{a p}(t)=\frac{1}{2}$ for almost all $t \in[0,1]$ and $\operatorname{dim}_{\mathrm{H}} \operatorname{graph} Z=$ $\underline{\operatorname{dim}}_{\mathrm{B}} \operatorname{graph} Z=\overline{\operatorname{dim}}_{\mathrm{B}} \operatorname{graph} Z=1 \frac{1}{2}$.

Proof. This is immediate from Theorems 5.3 and 5.4, since by Proposition 3.1 index- $\frac{1}{2}$ fBm has the maximum property.

\section{Synthesis of fractional Brownian surfaces}

The remainder of this paper is devoted to numerical investigation of the maximum conjecture and the fractal characteristics of the horizon function (box dimension and Hölder exponent). To this end, we have synthesized a large number of traces of fBm-s and fBf-s. In this section we describe the methods used for generating sample paths for various values of $\alpha$, and present some graphs. Section 7 explains the procedures for investigating the maximum property. Sections 8 and 9 are devoted to the estimation of the box dimension and Hölder exponent of the horizon $Z$.

Several methods have been proposed for synthesizing fractional Brownian motion, and, more generally, fractional Brownian fields. They include Choleski decomposition, midpoint displacement and its various improvements (Daniel \& Willski 1977), spectral synthesis (Kaplan \& Kuo 1996), wavelet-based methods, and synthesis based on differential models (Kesner 1974). The reason why so many algorithms have appeared is that synthesizing fBm-s is by no means an easy process, especially if one needs to build large traces. The problem lies mainly in the non-Markovian nature of $\mathrm{fBm}$, the strong correlations that it displays (for $\alpha<1 / 2$, each increment is negatively correlated with all the others, while for $\alpha>1 / 2$ the spectral density of the increments is singular at the origin). Though the Choleski method allows exact synthesis, a plain implementation suffers from two drawbacks: a large amount of computing time and a large amount of memory are needed to construct the signal. Under some minor restrictions on the generated traces, it is possible to use fast and efficient algorithms for Choleski decomposition, but various approximate methods using different approaches have also been designed that allow reasonable computer time/memory requirements. 
We first describe in some detail the principal steps involved in the classical Choleski decomposition method. Assume we wish to generate samples of an index- $\alpha \mathrm{fBm} X$ at $N$ equidistant points of $[0,1]$. Let $D X$ denote the discrete increments of $X$, that is $D X(k / N)=X(k / N)-X((k-1) / N), k=1, \ldots N$. These increments form a discrete (strict sense) stationary Gaussian process with zero mean, and the statistical properties of the vector $D X_{N}=(D X(1 / N), D X(2 / N), \ldots, D X(1))$ are entirely determinated by the autocovariance matrix $A_{N}=\mathrm{E}\left(D X_{N}\left(D X_{N}\right)^{T}\right)$, where $U^{T}$ denotes the transpose of $U$. From (2.1), $\mathrm{E}(D X(i / N) D X((i+k) / N))=\frac{1}{2 N^{2 \alpha}}\left(|k+1|^{2 \alpha}+|k-1|^{2 \alpha}-2|k|^{2 \alpha}\right)$. Since $A_{N}$ is positive definite, it may be written using its Choleski decomposition as:

$$
A_{N}=L_{N} L_{N}^{T}
$$

where $L_{N}$ is an invertible lower triangular matrix. Let $D Y_{N}=(D Y(1 / N), D Y(2 / N), \ldots, D Y(1))$ be an $N$ samples realization of a unit variance centered white Gaussian noise. It is easy to see that the autocovariance matrix of the random vector $L_{N} D Y_{N}$ is exactly $A_{N}$. Indeed:

$$
\mathrm{E}\left(L_{N} D Y_{N}\left(L_{N} D Y_{N}\right)^{T}\right)=L_{N} \mathrm{E}\left(D Y_{N}\left(D Y_{N}\right)^{T}\right) L_{N}^{T}=A_{N}
$$

We may thus set $D X_{N}=L_{N} D Y_{N}$, and generate a realization of the index- $\alpha$ fBm $X$ as $X(k / N)=\sum_{p=1}^{k} D X(p / N)$. Since $A_{N}$ depends only on $N$ and $\alpha$, it is entirely determined once we have fixed the exponent and the number of points we wish to generate. The problem of synthetizing a sample of an $\mathrm{fBm}$ is thus reduced to that of computing $L_{N}$ from $A_{N}$. Note that, so far, we have only used the fact that $A_{N}$ is a valid autocovariance matrix, so that it has a Choleski decomposition. Thus the procedure above may be applied for synthetizing any discrete Gaussian process. However, a direct method for general Choleski factorization has complexity $O\left(N^{3}\right)$ and requires $O\left(N^{2}\right)$ memory. This precludes the use of this approach for building large traces. Fortunately, when the process is stationary (this is why we work with $D X$ rather than $X$ ) and in the common case where the samples are equi-spaced, the matrix considered is Toeplitz: one can then use fast algorithms, such as the Schur or Levinson algorithms, which have complexity $O\left(N^{2}\right)$ and need $O(N)$ memory. It is possible to do even better if one forces $N$ to be a power of 2. Since such a requirement should not affect the verification of the maximum property conjecture, we shall restrict ourselves to this case. Then, the doubling Schur algorithm (Ammar \& Gragg 1988) allows the complexity to be reduced to $O\left(N\left(\log _{2}(N)\right)^{2}\right)$. This is very reasonable and permits the synthesis of quite large traces.

We now turn to the oldest of the approximate algorithms, the midpoint displacement. In the case of Brownian motion, this is in fact the original construction by Paul Lévy of the Wiener process. This method is very effective, as its complexity is linear in the number of samples. When $\alpha$ differs from $1 / 2$, however, it is no longer exact, and the resulting process, while still Gaussian, has second order properties that differ significantly from those of the fBm. In (Daniel \& Willski 1977), an improvement of the classical scheme is proposed, which allows one to recover approximately the right covariance function with a low computational burden. The algorithm is based on the notion of a multiresolution process, and improves on the classical method by using statistical descriptions of both the interpolation and displacement steps.

Wavelet based methods rely mainly on the fact that the wavelet transform acts as a 'whitening filter' on fractional Brownian motion. This allows easy synthesis of the wavelet coefficients of $\mathrm{fBm}$. However, the problem of building the low frequency approximation 
of the signal remains, and, while this methods allow synthesis of large datasets, it does not appear to be exact enough for our purposes.

An interesting spectral method for generating an approximation to a two dimensional $N \times N$ fBf is the incremental Fourier synthesis described in (Kaplan \& Kuo 1996). The idea is to create first a periodic random field of size $2 N \times 2 N$ with statistics close to those of the increments of the $\mathrm{fBf}$ over half the spatial period. Periodic random fields are easy to generate because their Karhuenen-Loève transform is simply the two dimensional discrete Fourier transform. The approximate $\mathrm{fBf}$ is then obtained by adding up the increments. Thanks to the use of the fast Fourier transform, the complexity of this method is only $O\left(N^{2} \log _{2}(N)\right)$ for a $N \times N$ image, with a memory requirement of $O\left(N^{2}\right)$. An alternative spectral method that gives an approximation to fBf but has complexity $O(N \log N)$ is described in (Yin 1996).

Finally, while methods based on differential models (that is where the process is obtained as a solution of a stochastic differential equation) are interesting because they have a physical meaning, they do not seem to yield correct approximations to $\mathrm{fBm}$. In fact, these methods are mainly used to study generic ' $1 / f$ ' noises.

Since we wish to test numerically a conjecture related to a very fine property of fractional Brownian motion, it is important to ensure that the synthesized traces are as close as possible to real sample paths of fBm-s. Thus we have chosen to use the only exact method, i.e. the Choleski decomposition via the doubling Schur algorithm. This implementation allowed us to generate traces of fBm with 131072 sample points and $2048 \times 2048 \mathrm{fBf}$. More specifically, the following data were synthesized:

- verification of the maximum property: for each value of $\alpha=0.1,0.2, \ldots, 0.9,100$ traces of fBm with 131072 sample points were generated.

- estimation of the box dimension and Hölder exponent of the horizon function: for each value of $\alpha=0.1,0.2, \ldots, 0.9,100$ traces of fBf with $2048 \times 2048$ sample points were generated, from which horizons with 2048 sample points were obtained.

In addition, two other kinds of traces were synthesized:

- fBm with 131072 sample points and fBf with $2048 \times 2048$ sample points generated with the spectral synthesis method.

- fBm with 262144 sample points and fBf with $4096 \times 4096$ sample points generated again with the spectral synthesis method.

This provided a test for possible discrepancies depending on the synthesis method and on the size of the samples. In all cases, to within statistical fluctuations, the same results were obtained on the three kinds of traces. It is worth recalling that the maximum property and thus the fractal estimates are known to hold for the Brownian motion (with $\alpha=\frac{1}{2}$ ), see Corollary 5.5, so index $\frac{1}{2} \mathrm{fBf}$ gives a way to assess the validity of our synthesis and estimation procedures. Figure 1 presents some realisations of fBf with $\alpha=0.1,0.3,0.5,0.7,0.9$ and $2048 \times 2048$ sample points. (Note that, although $X(0,0)=0$, we have renormalised the colour scale in each case to make use of the whole colour range.) Superimposed is the function $U_{t}$ : the black dots are the points where $X(t, u)$ attains a maximum w.r.t. to $u$. It is interesting to note how these points are distributed. Whilst Proposition 5.1 should be satisfied, $U_{t}$ has many of discontinuities, somewhat reminiscent of a Lévy process. Figure 2 shows the corresponding horizon functions $Z_{t}$. 
Since we are always dealing with fBm (resp. fBf) defined on $[0,1]$ (resp. $[0,1] \times$ $[0,1])$, the number of samples must be interpreted as the inverse of the resolution. For instance, an fBm with 131072 sample points requires the following points to be generated: $X(1 / 131072), X(2 / 131072), \ldots, X(131071 / 131072), X(1)$. This is particularly important in testing the maximum property.

\section{$7 \quad$ Numerical verification of the maximum conjecture}

In this section we test the maximum Conjecture 3.2, that is whether for all fixed $\alpha \in(0,1)$ and all $\epsilon>0$, there exists a.s. $\delta=\delta(\alpha, \epsilon)>0$ such that:

$$
X(t) \leq X\left(t_{m}\right)-\left|t_{m}-t\right|^{\alpha+\epsilon} \text { for all } t \in[0,1] \text { with }\left|t_{m}-t\right| \leq \delta .
$$

where $X$ is index- $\alpha \mathrm{fBm}$ and $t_{m}$ is the point where $X$ attains its maximum. As a qualitative test, we provide the following displays:

- Figure 3 shows traces of fBm with $\alpha=0.1,0.3,0.5,0.7,0.9$ and 131072 sample points, along with the corresponding curves $Y(t)=X\left(t_{m}\right)-\left|t_{m}-t\right|^{\alpha+\epsilon}$, with $\epsilon=0.05$ (as the case of Brownian motion is of special interest, we show two realizations with $\alpha=0.5)$. Notice that, whilst in the majority of cases, the graph is only locally below the theoretical envelope (as expected), for several realizations, $Y(t)$ is a tight upper bound for the whole path,

- Figure 4 shows zooms of the traces of Figure 3 around the maxima in the cases of $\alpha=0.1,0.5,0.9$, again with the $Y(t)$ curves. In all cases, the path of $X$ falls nicely below the envelope in the neighbourhood of $t_{m}$,

- Figure 5 gives a zoom around the maximum of an $\mathrm{fBm}$ with $\alpha=0.5$ along with both the upper envelope $Y(t)$ and the lower theoretical envelope $V(t)=X\left(t_{m}\right)-\left|t_{m}-t\right|^{\alpha-\epsilon}$ corresponding to the Hölder regularity of $X(t)$. It may be seen that, for a given $\epsilon$, the neighbourhood corresponding to the validity of the lower bound is smaller than the one for the upper bound. This property was observed on most traces.

In order to perform quantitative tests, we used the traces generated by the Choleski method described above. Thus, for each value of $\alpha$, we produced 100 sample paths with 131072 points. The difficulty in obtaining a meaningful verification is that equation (7.1) does not tell us how $\epsilon$ and $\delta$ should be chosen. It could be that for a given $\epsilon$, say 0.05 , the corresponding $\delta$ is so small that traces much larger (i.e. with a much higher resolution) than the ones that were synthesised are needed. Conversely, if we choose $\epsilon$ large enough and $\delta$ small enough, even if $X$ does not satisfy the maximum property, equation (7.1) is likely to hold for this particular choice of $(\epsilon, \delta)$. We adopted the following strategy. For each value of $\alpha$, and for each $\epsilon=0.02,0.04, \ldots, 0.1$, we computed $\delta(\alpha, \epsilon)$ as the largest number such that, in at least $95 \%$ of the cases (that is for at least 95 traces out of the 100 ones available for each $\alpha$ ), equation (7.1) holds. In other words, the value of $\delta$ selected is such that, "almost surely", all points in a $\delta$-neighbourhood of the maximum fall under the conjectured envelope, while this property fails for any larger neighbourhood.

Table 1 shows the results for the 500 traces corresponding to $\alpha=0.1,0.3,0.5,0.7,0.9$. Note that $\delta$ is expressed in absolute units (not as a number of samples) obtained from the 
ratio (number of samples)/131072. The same tests were performed on the traces with the remaining values of $\alpha$ and also on the sample paths obtained with the spectral synthesis method (both resolutions), yielding essentially the same behaviour.

In view of the results in Table 1 , it is reasonable to conclude that the numerical simulations do confirm the maximum conjecture. Firstly, even for values of $\epsilon$ as small as $1 / 45$ of $\alpha$ (for $\alpha=0.9$ ) and $1 / 5$ of $\alpha$ (for $\alpha=0.1$ ), there exists a neighbourhood such that, for at least $95 \%$ of the realizations, the expected inequality holds. This neighbourhood has at least size 0.01 (corresponding roughly to 1300 samples points) or $1 \%$ of the size of the path, and in some case this size increases to 0.03. Secondly, Table 1 reveals a nice evolution of the size of the neighbourhood with respect to $\epsilon$ : for low values of $\alpha$, the "discontinuous" increase of $\delta$ matches the antipersistant property of the paths, whilst for large values of $\alpha$, the increase in $\delta$ is smooth paralleling the long range dependance in the realizations. Finally, recalling that the result holds true for $\alpha=0.5$, we see that essentially the same things happen for all values of the exponent. Of particular interest is the fact that, for all values of $\epsilon$ except 0.08 , the largest neighbourhood sizes are not attained in the case of usual (index- $\frac{1}{2}$ ) Brownian motion. Thus, according to the numerical experiments, there does not seem to be anything special about $\alpha=\frac{1}{2}$.

Note that all the tests and comments above make a hidden assumption. Given a random element $\omega$ of the sample space, that is for a fixed realization $X_{t}(\omega)$ of an fBm on $[0,1]$, we first perform a sampling to get the points $X_{t_{1}}(\omega), \ldots, X_{t_{n}}(\omega)$, and then find the maximum $\left(t_{m}, X_{t_{m}}\right)$ over this finite set of samples. However, depending on $n$, it might well be that $t_{m}$ bears little relationship to the location of the "true" maximum $t^{*}$, that is the maximum of $X_{t}$ over $[0,1]$. One way around this is as follows. Knowing the Hölder exponent $\alpha$ of $X_{t}$ and its $\alpha$-Hölder norm, both of which are estimated by (2.3), it is possible to construct from $X_{t_{1}}(\omega), \ldots, X_{t_{n}}(\omega)$ a continuous path $\overline{X(t)}$ that is an upper bound to $X_{t}$ for all $t$. If $X_{t_{m}}$ is larger than $\overline{X(t)}$ for all $t$ smaller than $t_{m-1}$ and larger than $t_{m+1}$, then we are assured that the true maximum lies in $\left[t_{m-1}, t_{m+1}\right]$, with a value $X\left(t^{*}\right)$ "close" to $X_{t_{m}}$, and this is the best we can achieve. Otherwise, $t^{*}$ might be located arbitrarily far from $t_{m}$. This last situation may for instance occur in figure 13 . In this case, the numerical verification of the maximum conjecture is meaningless, since the maximum is not well defined on the sample path. In our experiments, we discarded such traces. For more on the problem of locating the maximum of a Hölder function from a sampling (see Lutton et al 1998).

\section{Estimation of the box dimension of the horizon}

In this section we present numerical evidence to support Theorem 5.3, in particular that the box dimension of the horizon is equal to $2-\alpha$ for an index- $\alpha \mathrm{fBf}$.

There are various methods for estimating the box dimension of a graph $F$. The crudest one, which simply consists in counting the number of boxes $N(\epsilon)$ of a given size $\epsilon$ that intersect $F$ and then making a linear regression of $\log (N(\epsilon))$ against $\log (\epsilon)$ suffers from many well-known defects, and does not in general give good results, so more refined procedures are needed. The first procedure we used is the variation method (Tricot 1995). Define the $\epsilon$-variation of $Z$ :

$$
\operatorname{var}_{Z}(\epsilon)=\int_{0}^{1} \operatorname{osc}_{Z}(t, \epsilon) d t
$$


where $\operatorname{osc}_{Z}(t, \epsilon)$ is the oscillation of $Z$ in the ball centered at $t$ with radius $\epsilon$,

$$
\operatorname{osc}_{Z}(t, \epsilon)=\sup _{t-\epsilon<u<t+\epsilon} Z(u)-\inf _{t-\epsilon<u<t+\epsilon} Z(u)
$$

Then, assuming that the box dimension exists (that is $\underline{\operatorname{dim}}_{\mathrm{B}} \operatorname{graph} Z=\overline{\operatorname{dim}}_{\mathrm{B}} \operatorname{graph} Z=$ $\operatorname{dim}_{\mathrm{B}} \operatorname{graph} Z$ ), we have

$$
\operatorname{dim}_{\mathrm{B}} \operatorname{graph} Z=\max \left\{1, \lim _{\epsilon \rightarrow 0}\left(2-\frac{\log \left(\operatorname{var}_{Z}(\epsilon)\right)}{\log (\epsilon)}\right)\right\}
$$

see [5, Section 11.1], so $\operatorname{dim}_{\mathrm{B}}$ graph $Z$ may be estimated as a regression of $\frac{1}{\epsilon^{2}} \log \left(\operatorname{var}_{Z}(\epsilon)\right)$ vs. $|\log \epsilon|$. The main advantage of this procedure compared to the box method is that it is invariant under a change of scale. It also allows for a smooth evolution of the window size $\epsilon$ and leads to a fast implementation. Its well-known drawback is that oscillations are sensitive to noise, but this is not a problem in our setting.

A second estimator was examined, based on the computation of the regularization dimension $\operatorname{dim}_{\mathrm{R}}$, defined as follows. Let $\chi(t)$ be a kernel function of Schwartz class $\mathcal{S}$ such that

$$
\int \chi=1
$$

Let $\chi_{a}(t)=\frac{1}{a} \chi\left(\frac{t}{a}\right)$ be the dilation of $\chi$ at scale $a>0$ and $Z^{a}$ be the convolution of $Z$ with $\chi_{a}$. Since $Z^{a} \in \mathcal{S}$, the length $\Lambda_{a}$ of its graph is finite. The regularization dimension of graph $Z$ is defined as

$$
\operatorname{dim}_{\mathrm{R}} \operatorname{graph} Z=1+\varlimsup_{a \rightarrow 0} \frac{\log \left(\Lambda_{a}\right)}{-\log a}
$$

Thus $\operatorname{dim}_{R}$ measures the speed at which the length of decreasingly regularised versions of a fractal graph tends to infinity. It is easy to check that, for a continuous curve $C$, one always has

$$
\operatorname{dim}_{\mathrm{H}} C \leq \operatorname{dim}_{\mathrm{R}} C \leq \underline{\operatorname{dim}}_{\mathrm{B}} C
$$

Thus, if the maximum property is true, we should have that $\operatorname{dim}_{\mathrm{R}}$ graph $Z$ coincides with the common value of the box and Hausdorff dimensions of the horizon $Z$. The advantage of using $\operatorname{dim}_{R}$ is that it leads to a more robust estimator than other methods.

The results obtained with the two methods on the 900 traces generated through Choleski decomposition are displayed in Table 2. For each value of $\alpha$ and both estimators, the mean value and the standard deviation as measured on the 100 corresponding traces are shown. Once again, tests on horizon functions obtained through spectral synthesis yielded similar behaviour. Note that in all tests that consider the whole path, one hundred points at each end of the sample were discarded to avoid boundary problems.

Several conclusions may be drawn from Table 2. Firstly, both estimators agree, within statistical fluctuations, for all values of $\alpha$. Since they are based on different procedures, this gives us confidence that the results are meaningful. Secondly, all the means are close to the predicted values (the largest error is 0.05 for the variation method and 0.04 for the regularization dimension), well within error bars given by the standard deviation. Thirdly, the values obtained for both the means and the standard deviations are of the order of what is usually reported in the litterature when testing the performances of new estimators of the box dimension on classical "fractal" curves such as fBm or Weierstrass functions. Recalling that such curves are self-similar while the horizon has no direct scale 
invariance properties gives even more confidence in the results. Finally, note that better results are obtained for medium range values of the box dimension, while low values are overestimated and large values are underestimated. Again, this is common in box dimension estimation, with similar behaviour observed in almost all situations. While methods exist to correct such bias, they usually make assumptions not applicable here. The estimate for $\alpha=0.5$, where the theoretical value is known to be 1.5 , is no better than for the neighbouring values $\alpha=0.3,0.4,0.6$ and 0.7 . This shows once again that there does not seem to be anything special about $\alpha=0.5$. In summary, Table 2 clearly supports the parts of Theorem 5.3 pertaining to box dimension.

\section{Estimation of Hölder exponents of the horizon}

In this section we estimate the Hölder exponent $\underline{H}_{Z}(t)$, given by (1.2), of the horizon function $Z$, and give numerical evidence to support the conclusion of Theorem 5.4. From the definition, it is obvious that such a quantity is extremely difficult to estimate from numerical data, and we describe various steps that simplify the computation.

First, it is easy to see that (1.2) is equivalent to

$$
\underline{H}_{Z}(t)=\liminf _{\epsilon \rightarrow 0^{+}} \frac{\log \operatorname{osc}_{Z}(t, \epsilon)}{\log \epsilon} .
$$

The form (9.1) leads to better estimates as it takes more data into account in the computation: instead of involving the values $Z(u)-Z(t)$ where only $u$ varies in the neighbourhood of $t$, it depends on $Z(u)-Z(v)$ for all $(u, v)$ in the neighbourhood of $t$.

The second step is to eliminate the lower limit in the definition of $\underline{H}_{Z}(t)$. Indeed, estimating a lower limit from numerical data means that we are able to decide which neighbourhoods should be taken into account and which should be discarded. Without any further information on the behaviour of $\operatorname{osc}_{Z}(t, \epsilon)$, this is a very hard task. Fortunately, we may invoke Theorem 5.4, which yields a fine measure theoretic property of $\underline{H}_{Z}(t)$, much stronger that the basic definition. Indeed, the proofs of Corollary 4.3 and Theorem 5.4 imply that, for almost all sequences of $\epsilon$ convergent to 0 at a geometric rate, we should obtain the right exponent. We may thus replace the lower limit by a plain limit, which we evaluate classically as a regression of $\log \operatorname{osc}_{Z}(t, \epsilon)$ vs. $\log \epsilon$.

Thirdly, we shall use a quantity which is closely related to the pointwise Hölder exponent $\underline{H}_{Z}(t)$, termed the local Hölder exponent, and defined as

$$
\underline{H}_{Z}^{l}(t)=\liminf _{\epsilon \rightarrow 0^{+}} \frac{\log \operatorname{osc}_{Z}(t, \epsilon)}{\log \left|t_{1}-t_{2}\right|} .
$$

where $\left(t_{1}, t_{2}\right)$ is a pair of extreme points of the oscillation. Then $\underline{H}_{Z}^{l}$ is a more 'stable' function of $t$ that $\underline{H}_{Z}$, and is thus easier to estimate. This may be seen by noting that, while $\underline{H}_{Z}^{l}$ has to be a lower semi-continuous function, $\underline{H}_{Z}$ need only be a lower limit of a sequence of continuous functions (Guiheneuf \& Lévy Véhel 1998). We thus expect a less erratic behaviour for the local exponent. Further, though $\underline{H}_{Z}$ and $\underline{H}_{Z}^{l}$ are not in general equal, they should coincide in our case: if the maximum property holds, $\underline{H}_{Z}(t)$ must, a.s., equal $\alpha$ for almost all $t$, implying that $\underline{H}_{Z(t)}^{l}$ will also a.s. equal $\alpha$ for almost all $t$.

As before, we performed tests on the 900 traces generated by the Choleski method. The results are displayed in Table 2. For each value of $\alpha$, the mean value and the standard 
deviation of $\underline{H}_{Z}$ as measured from 100 traces are given. Note that we are dealing here with two averaging procedures: one on all realizations and one along the path of each particular realization. Table 4 shows what happens if we consider only one particular sample path. For each $\alpha$, one horizon was chosen at random, and the mean value and standard deviation of the function $\underline{H}_{Z}(t)$ was computed. Conversely, Table 3 displays the result obtained on averaging, for a fixed $t_{0}$, the values of $\underline{H}_{Z}\left(t_{0}\right)$ on all 100 realizations.

The conclusions of this numerical study parallel to a certain extent those of the preceeding section. However, due to its local nature, it is obvious that estimating a Hölder exponent is a much harder task than estimating a box dimension. In particular, the sampling has more influence: in general, it entails two competing effects that bias the estimation. Firstly, the oscillation as measured on the discrete data is always a lower bound for the "true" oscillation. This effect is dominant when $\underline{H}_{Z}(t)$ is small, because the discrepancy will be larger, and results in an overestimate of $\underline{H}_{Z}(t)$. For large values of $\underline{H}_{Z}(t)$, an "interference" phenomenon occurs, that is the oscillations around a "smooth" point $t$ may be dominated by what happens in a smaller neighbourhood of an "irregular" point close to $t$. This translates into an underestimate of $\underline{H}_{Z}(t)$. These heuristic explanations can be made precise under certain assumptions of scale invariance, these precise arguments are not available for the horizon, and we must be content with the expectation that $\underline{H}_{Z}(t)$ will be overestimated for small values and underestimated for large values, a fact borne out in Tables 3, 4 and 5. The difficulty in estimating $\underline{H}_{Z}(t)$ is reflected in our tables by the relatively large values of standard deviation, especially when dealing with an average over one particular realization. This is not unexpected since this averaging procedure does not have a real statistical basis. Table 5 gives somewhat better resuls, although the biases are still large for extreme values of $\underline{H}_{Z}(t)$, and the standard deviations are quite big. Note that in the three tables, the worst results are obtained for low values of $\underline{H}_{Z}(t)$, possibly indicating that in our case, the discrepancy between the measured oscillation and the true one dominates the effect of interference. Finally, the results for $\alpha=0.5$ are again not very different from those of neighbouring values of the exponent, an argument in favour of the conjectured results. In conclusion, though the numerical estimates for the Hölder exponents are not as sharp as for the box dimensions, they do not discredit the conclusions of Theorem 5.4. Indeed, for $\alpha=0.4,0.6$ and 0.7 , they match the theoretical predictions fairly closely.

\section{Conclusion}

The work in this paper gives strong support to the horizon conjecture, that the horizon of an index- $\alpha$ fractional Brownian surface almost surely has the same Hölder exponents as the surface itself, with the box and Hausdorff dimensions of the horison one less than those of the surface. We have proved this rigorously for the usual index- $\frac{1}{2}$ Brownian surface, and also for the index- $\alpha$ Brownian surface for $0<\alpha<1$ assuming the maximum property for index- $\alpha$ Brownian functions. For all $\alpha$ we have presented substantial computational evidence both for the horizon conjecture for index- $\alpha$ Brownian surfaces and for the maximum conjecture for the index- $\alpha$ Brownian functions. 


\section{References}

Addison, P.S. \& Ndumu, A.S. 1999 Engineering applications for fractional Brownian motion: self-affine and self-similar random processes. Fractals 7, 151-157.

Adler, R.J. 1981 The Geometry of Random Fields. John Wiley.

Ammar, G.S. \& Gragg W.B. 1988 Superfast solution of real positive definitive Toeplitz systems. SIAM J. Matrix Anal. Appl. 9, 61-76.

Barlow, M.T. \& Perkins. E. 1984 Levels at which every Brownian excursion is exceptional, in Lecture Notes in Math. 1059, 1-28. Springer .

Daniel, M.M. \& A.S. Willski. 1977 Modeling and estimation of fractional Brownian motion using multiresolution stochastic processes, in Fractals in Engineering Lévy Véhel, J., Lutton E. \& Tricot, C. Eds., 124-137. Springer.

Falconer, K.J. 1990 Fractal Geometry - Mathematical Foundations and Applications. John Wiley.

Falconer, K.J. 1991 The horizon problem for random surfaces. Math. Proc. Cambridge Philos. Soc. 109, 211-219.

Geman, D. \& Horowitz, J. 1980 Occupation densities. Ann. Probab. 8, 1-67.

Guiheneuf, B \& Lévy Véhel, J. 1998 2-Microlocal analysis and application in signal processing, International Wavelets Conference, Tangier, 27-39.

Itô, K. \& McKean, H.P. 1974 Diffusion Processes and their Sample Paths. SpringerVerlag.

Jaggard, D.L. 1991 Fractal Electrodynamics and Modeling, in Directions in Electromagnetic Wave Modeling, H. L. Bertoni \& L. B. Felsen, eds., 435-446. Plenum Publishing Co.

Kahane J.-P. 1985 Some Random Series of Functions. Cambridge University Press.

Kaplan, L.M. \& Kuo C.C.J. 1996 An improved method for 2-D self-similar image synthesis. IEEE Tr. Image Proc. 5, 754-761.

Kesner, M.S. 1974 1/f noises. Proc. IEEE 70, 212-218.

Lundahl, T., Ohley, W.J., Kay, S.M. \& Siffert, R. 1986 Fractional Brownian motion : a maximum likelihood estimator and its application to image texture. IEEE Tr. Med. Imaging MI-5(3), 152-161.

Lutton E. \& Lévy Véhel, J. 1998 Hölder functions and Deception of Genetic Algorithms. IEEE Tr. Evolutionary Computing 2, 56-71.

Mandelbrot, B.B. 1982 The Fractal Geometry of Nature. W.H. Freeman.

Mandelbrot B.B. \& Van Ness, J. 1968 Fractional brownian motion, fractional noises and applications. Siam Review 10, 422-437.

Peitgen, H.-O. \& Saupe, D. 1988 The Science of Fractal Images. Springer Verlag.

Pitt, L.D. 1978 Local times for Gaussian vector fields. Indiana Univ. Math. J. 27, 309-330.

Tricot, C. 1995 Curves and Fractal Dimension. Springer Verlag.

Williams, D. 1974 Path decomposition and continuity of local times for one-dimensional diffusions I. Proc. London Math. Soc.(3) 28, 738-768.

Yin. 1996 New Methods for Simulation of Fractional Brownian Motion. J. Comput. Phys. 127, 66-72. 


\begin{tabular}{||c|c|c|c|c|c|c||}
\hline \hline & $\epsilon$ & 0.02 & 0.04 & 0.06 & 0.08 & 0.1 \\
\hline$\alpha=0.1$ & $\delta$ & 0.03 & 0.05 & 0.05 & 0.05 & 0.05 \\
\hline$\alpha=0.3$ & $\delta$ & 0.03 & 0.05 & 0.05 & 0.07 & 0.08 \\
\hline$\alpha=0.5$ & $\delta$ & 0.02 & 0.03 & 0.05 & 0.08 & 0.08 \\
\hline$\alpha=0.7$ & $\delta$ & 0.01 & 0.02 & 0.03 & 0.04 & 0.05 \\
\hline$\alpha=0.9$ & $\delta$ & 0.02 & 0.025 & 0.03 & 0.033 & 0.04 \\
\hline \hline
\end{tabular}

Table 1: Evolution of the size of the neighbourhood where the maximum property is valid.

\begin{tabular}{|c|c|c|c|c|c|c|c|c|c|c|c|c|c|c|c|c|c|c|}
\hline$\alpha:$ & \multicolumn{2}{|c|}{0.1} & \multicolumn{2}{|c|}{0.2} & \multicolumn{2}{|c|}{0.3} & \multicolumn{2}{|c|}{0.4} & \multicolumn{2}{|c|}{0.5} & \multicolumn{2}{|c|}{0.6} & \multicolumn{2}{|c|}{0.7} & \multicolumn{2}{|c|}{0.8} & \multicolumn{2}{|c|}{0.9} \\
\hline & $\mathrm{m}$ & std & $\mathrm{m}$ & std & $\mathrm{m}$ & std & $\mathrm{m}$ & std & $\mathrm{m}$ & std & $\mathrm{m}$ & std & $\mathrm{m}$ & std & $\mathrm{m}$ & std & $\mathrm{m}$ & std \\
\hline Variation Method & 1.87 & .07 & 1.76 & .09 & 1.71 & .07 & 1.62 & .05 & 1.47 & .06 & 1.38 & .07 & 1.29 & .06 & 1.17 & .07 & 1.15 & .08 \\
\hline $\begin{array}{l}\text { Regularization } \\
\text { Dimension }\end{array}$ & 1.88 & .09 & 1.81 & .06 & 1.69 & .08 & 1.62 & .06 & 1.49 & .05 & 1.4 & .06 & 1.31 & .07 & 1.23 & .07 & 1.14 & .09 \\
\hline
\end{tabular}

Table 2: Mean $(\mathrm{m})$ and standard deviation (std) of the estimators for the box dimension at the horizon.

\begin{tabular}{|c|c|c|c|c|c|c|c|c|c|c|c|c|c|c|c|c|c|c|}
\hline $\bar{~} \overline{\alpha:}$ & \multicolumn{2}{|c|}{$\overline{0.1}$} & \multicolumn{2}{|c|}{0.2} & \multicolumn{2}{|c|}{0.3} & \multicolumn{2}{|c|}{$\overline{0.4}$} & \multicolumn{2}{|c|}{$\overline{0.5}$} & \multicolumn{2}{|c|}{$\overline{0.6}$} & \multicolumn{2}{|c|}{$\overline{0.7}$} & \multicolumn{2}{|c|}{$\overline{0.8}$} & \multicolumn{2}{|c|}{$\overline{0.9}$} \\
\hline & $\mathrm{m}$ & std & $\mathrm{m}$ & std & $\mathrm{m}$ & std & $\mathrm{m}$ & std & $\mathrm{m}$ & std & $\mathrm{m}$ & std & $\mathrm{m}$ & std & $\mathrm{m}$ & std & $\mathrm{m}$ & std \\
\hline $\begin{array}{l}\text { Estimated Hölder } \\
\text { exponent }\end{array}$ & 0.16 & 0.12 & 0.26 & 0.15 & 0.34 & 0.13 & 0.42 & 0.11 & 0.52 & 0.1 & 0.59 & 0.13 & 0.72 & 0.12 & 0.84 & 0.15 & 0.86 & 0.15 \\
\hline
\end{tabular}

Table 3: Estimated Hölder exponent at the horizon averaged over all positions and realizations.

\begin{tabular}{|c|c|c|c|c|c|c|c|c|c|c|c|c|c|c|c|c|c|c|}
\hline$\alpha$, & \multicolumn{2}{|c|}{0.1} & \multicolumn{2}{|c|}{0.2} & \multicolumn{2}{|c|}{0.3} & \multicolumn{2}{|c|}{0.4} & \multicolumn{2}{|c|}{0.5} & \multicolumn{2}{|c|}{0.6} & \multicolumn{2}{|c|}{0.7} & \multicolumn{2}{|c|}{0.8} & \multicolumn{2}{|c|}{0.9} \\
\hline & $\mathrm{m}$ & std & $\mathrm{m}$ & std & $\mathrm{m}$ & std & $\mathrm{m}$ & std & $\mathrm{m}$ & std & $\mathrm{m}$ & std & $\mathrm{m}$ & std & $\mathrm{m}$ & std & $\mathrm{m}$ & std \\
\hline $\begin{array}{l}\text { Estimated Hölder } \\
\text { exponent }\end{array}$ & 0.21 & 0.2 & 0.3 & 0.19 & 0.36 & 0.19 & 0.47 & 0.15 & 0.54 & 0.13 & 0.63 & 0.17 & 0.67 & 0.19 & 0.81 & 0.2 & 0.83 & 0.18 \\
\hline
\end{tabular}

Table 4: Estimated Hölder exponent at the horizon averaged over one realization.

\begin{tabular}{|c|c|c|c|c|c|c|c|c|c|c|c|c|c|c|c|c|c|c|}
\hline \multirow[t]{2}{*}{$\overline{\overline{\alpha:}}$} & \multicolumn{2}{|c|}{$\overline{0.1}$} & \multicolumn{2}{|c|}{$\overline{0.2}$} & \multicolumn{2}{|c|}{$\overline{00.3}$} & \multicolumn{2}{|c|}{$\overline{0.4}$} & \multicolumn{2}{|c|}{$\overline{0.5}$} & \multicolumn{2}{|c|}{$\overline{0.6}$} & \multicolumn{2}{|c|}{0.7} & \multicolumn{2}{|c|}{$\overline{0.8}$} & \multicolumn{2}{|c|}{$\overline{0.9}$} \\
\hline & $\mathrm{m}$ & std & $\mathrm{m}$ & std & $\mathrm{m}$ & std & $\mathrm{m}$ & std & $\mathrm{m}$ & std & $\mathrm{m}$ & std & $\mathrm{m}$ & std & $\mathrm{m}$ & std & $\mathrm{m}$ & std \\
\hline $\begin{array}{l}\text { Estimated Hölder } \\
\text { exponent }\end{array}$ & 0.15 & 0.18 & 0.25 & 0.17 & 0.33 & 0.18 & 0.39 & 0.12 & 0.49 & 0.1 & 0.57 & 0.15 & 0.72 & 0.13 & 0.83 & 0.15 & 0.87 & 0.17 \\
\hline
\end{tabular}

Table 5: Estimated Hölder exponent at the horizon averaged at a given position over all realizations. 